DOI 10.31489/2020No1/106-112

UDC: $533.682 ; 533.6 .01 ; 621.548$

\title{
MODELING AERODYNAMICS OF A WIND TURBINE WITH CYLINDRICAL BLADES IN A TURBULENT AIR FLOW
}

\author{
Sakipova S.E. ${ }^{1}$, Tanasheva N.K. ${ }^{2}$, Minkov L.L. ${ }^{3}$ \\ 1E.A. Buketov Karaganda State University, Karaganda, Kazakhstan, sesaule@mail.ru \\ 2Institute of Applied Mathematics, Karaganda, Kazakhstan, nazgulya tans@mail.ru \\ ${ }^{3}$ National Research Tomsk State University, Tomsk, Russia
}

\begin{abstract}
The article discusses some of the possibilities of computer simulation of aerodynamics flow past a three-bladed wind turbine using the ANSYS FLUENT software package. The peculiarity of the object of study is that the wind wheel consists of three blades rotating around its axis. The system of equations, boundary conditions and made during modeling assumptions are presented. The system of equations in approximating the $k-\varepsilon$ model of turbulence is solved by the finite volume method and applying the approach of multiple (nested) coordinate systems using the Ansys-Fluent package. The computational domain was divided into three types of subregions nested within each other. As a result of modeling the velocity field near the wind wheel surface vortex zones were visualized. It is showed that in the vicinity of the wind wheel central disk, the air flow unfolds in the opposite direction to the main flow. The dependences of aerodynamic coefficients on speed are obtained while the cylinders rotation speed varies in the range from $300 \mathrm{rpm}$ to $700 \mathrm{rpm}$.
\end{abstract}

Keywords: wind turbine, aerodynamics, rotating cylinder, ANSYS FLUENT software packages, drag force, lift force, Reynolds criterion, 3-dimensional modeling.

\section{INTRODUCTION}

It is known that to reduce energy security the country needs to deploy a number of renewable energy sources (RES), [1-4]. Statistics show that Central Asian countries rich in fossil fuels, in particular Kazakhstan, are leaders in the renewable energy segment, while countries with low hydrocarbon content have virtually no renewable energy facilities [3]. Despite economic difficulties in Kazakhstan, favorable conditions have been created for the development of renewable energy facilities. As was noted earlier [5-6], wind energy is the most relevant direction for the development of renewable energy sources, since wind power plants are one of the most environmentally friendly methods of energy production. In recent years, a number of domestic and foreign scientists and engineers have developed various original wind power plants, a detailed review of which is presented in [7-10]. An analysis of published works and well-known developments confirms that it is necessary to develop small or medium-sized autonomous wind farms that can efficiently convert wind energy into electrical or thermal energy.

The high level and capabilities of computational technology and 3D computer simulation sometimes allow us to replace complex experimental studies, for example, an aerodynamics of streamlined bodies. This does not exclude experiments in general, but it allows us not to interrupt the study, which is especially important in the absence of real possibilities for conducting practical tests, as at present in the quarantine period of forced self-isolation due to coronovirus. 3D computer modeling allows you to discover new aspects and important results that are very useful in practical implementation. In the present paper some aspects of computer simulation of the aerodynamics of a wind turbine with rotating cylinders using the ANSYS FLUENT software packages are discussed. Despite the fact that a cylindrical body has been used in aero-hydrodynamics for many years, the study and modeling of the aerodynamics of its flow under various conditions are still relevant. 
Indeed, the aerodynamics of the flow around a rotating cylinder depends not only on its surface state (smooth, porous, rough) and the shape of its ends (flat, rounded, cone-shaped, etc.), but also on its rotating speed around its axis [7, 11, 12]. For example, in [13] flows were compared over a smooth two-dimensional round cylinder and a finite round cylinder. In particular, the impact due to the textile roughness on the critical transition phenomenon was emphasized.

\section{Problem statement and system of equations.}

In paper [12] the problem was formulated at stage of solution in an axisymmetric statement, where the dependence on the azimuthal coordinate $\varphi$ was not taken into account. A computer model of wind turbines with three angled blades is also described and presented in detail. Fluid flow was assumed to be laminar and was described by a system of control equations in dimensional formulation, including: total energy conservation equation; momentum equation; continuity equation with corresponding boundary conditions. An unstructured computational grids of the model was generated in the ANSYS MESH subroutine, computations were performed in the ANSYS CFX and ANSYS FLUENT [14, 15]. Using these software packages a CAD-model of the wind turbine with rotating cylinders having flat disks at its ends has been developed. The results of a 3-D modeling of the dynamics of the aerodynamic drag force of a virtual three-bladed model of a wind turbine with rotating cylindrical blades allowed show in a first approximation, the physical flow pattern in the range of air flow variation from 3 to $10 \mathrm{~m} / \mathrm{s}$.

In this case, the following basic assumptions are made to describe the aerodynamics of the flow of air flowing around the rotating cylinders of a wind power plant.

1. The ratio of gas velocity to local sound velocity, $M \quad<0.1$. Due to the low values of the Mach numbers, the air flow can be described by equations valid for an incompressible medium.

2. Given condition 1 and due to insignificant temperature differences in the vicinity of the wind wheel, the flow can be considered isothermal.

3. Due to the high speeds of the incoming air flow, the Reynolds number Re> 104, at which the ratio of inertial forces to viscosity forces is large enough, the flow can be considered turbulent.

Let consider a system of equations describing the dynamics of the air flow around a wind wheel under given the above conditions.

$$
\begin{aligned}
& \frac{\partial u_{j}}{\partial x_{j}}=0, \\
& \frac{\partial \rho u_{i}}{\partial t}+\frac{\partial \rho u_{i} u_{j}}{\partial x_{j}}+\frac{\partial p}{\partial x_{i}}=\frac{\partial \tau_{i j}}{\partial x_{j}},
\end{aligned}
$$

where $\tau_{i j}=\left(\mu+\mu_{t}\right)\left[\frac{\partial u_{j}}{\partial x_{i}}+\frac{\partial u_{i}}{\partial x_{j}}\right]$ is the stress tensor.

When using the k- $\varepsilon$ turbulence model, the energy equation will be as follows

$$
\frac{\partial \rho k}{\partial t}+\frac{\partial \rho k u_{j}}{\partial x_{j}}=\frac{\partial}{\partial x_{j}}\left[\left(\mu+\frac{\mu_{t}}{\sigma_{k}}\right) \frac{\partial k}{\partial x_{j}}\right]+G_{k}-\rho \varepsilon .
$$

The equation of the specific dissipation rate of turbulent energy

$$
\frac{\partial \rho \varepsilon}{\partial t}+\frac{\partial \rho \varepsilon u_{j}}{\partial x_{j}}=\frac{\partial}{\partial x_{j}}\left[\left(\mu+\frac{\mu_{t}}{\sigma_{\varepsilon}}\right) \frac{\partial \varepsilon}{\partial x_{j}}\right]+\rho \varepsilon\left(C_{1} S-C_{2} \frac{\varepsilon}{k+\sqrt{v \varepsilon}}\right)
$$

The production of turbulent kinetic energy can be described as 


$$
G_{k}=\mu_{t} S^{2}
$$

where $S=\sqrt{2 S_{i j} S_{i j}}$ is a strain rate tensor module.

$$
C_{1}=\max \left(0.43, \frac{\eta}{\eta+5}\right), \quad \eta=S \frac{k}{\varepsilon}, \quad C_{2}=1.9
$$

Coefficient of turbulent viscosity

$$
\mu_{t}=\rho C_{\mu} \frac{k^{2}}{\varepsilon}
$$

where $C_{\mu}=\frac{1}{A_{0}+A_{s} \frac{k U^{*}}{\varepsilon}}, \quad U^{*}=\sqrt{S_{i j} S_{i j}+\Omega_{i j} \Omega_{i j}}, \quad \Omega{ }_{i j}=\overline{\Omega_{i j}}-\varepsilon_{i j k} \omega_{k}$.

$\overline{\Omega_{i j}}-$ vorticity tensor in a coordinate system moving with angular velocity.

The following values of constants and parameters are accepted in the calculations:

$$
\begin{aligned}
& A_{0}=4.04, \quad A_{s}=6 \cos (\phi), \quad \phi=\frac{1}{3} \cos ^{-1}(\sqrt{6} W), \quad W=\frac{S_{i j} S_{j k} S_{k i}}{\xi^{\natural}} \\
& \tilde{S}=\sqrt{S_{i j} S_{i j}}, \quad S_{i j}=\frac{1}{2}\left(\frac{\partial u_{i}}{\partial x_{j}}+\frac{\partial u_{j}}{\partial x_{i}}\right), \quad \Omega_{i j}=\frac{1}{2}\left(\frac{\partial u_{i}}{\partial x_{j}}-\frac{\partial u_{j}}{\partial x_{i}}\right)
\end{aligned}
$$

Here $\varepsilon_{i j k}$ are the components of the Levi-Civitt tensor.

Turbulent Prandtl numbers: $\sigma_{k}=1, \sigma_{\varepsilon}=1.2$.

The boundary conditions for describing the change in the turbulent kinetic energy on the wall are specified as follows

$$
\frac{\partial k}{\partial n}=0 \text {. }
$$

Directly, the value of the dissipation rate of turbulent kinetic energy $\varepsilon$ in the near-wall region is calculated from the condition that the kinetic energy production of turbulent pulsations and its dissipation are equal under the assumption of a logarithmic distribution of the average gas velocity.

$$
\varepsilon_{p}=\frac{C_{\mu}^{0.75} k_{p}^{1.5}}{\kappa y_{p}}
$$

where $\kappa=0.42 ; y_{p}$ is the distance from the center of the wall cell to the wall.

The value of $y_{p}$ can be determined as follows:

$$
y_{p}=\frac{\mu}{\rho C_{\mu}^{0.25} k_{p}^{0.5}} y^{*}, \quad y^{*}=11.225
$$

Boundary conditions at the entrance to the computational domain are

$$
\begin{aligned}
& u_{x}=0, u_{y}=0, u_{z}=V \\
& \varepsilon=C_{\mu}^{0.75} \frac{k^{1.5}}{0.07 D_{h}}, \quad k=\frac{3}{2}(V \cdot I)^{2}
\end{aligned}
$$

Here $D_{h}$ is the hydraulic size of the input section of the region, $D_{h}$ was taken equal to $1 \mathrm{~m}$; 
$\mathrm{I}-$ is the intensity of turbulent pulsations, $\mathrm{I}=0.1$;

Boundary conditions at the exit from the region: $p=p_{H}$;

Boundary conditions on the walls of the wind turbine: $u_{i}=U(t, x, y, z)$, where $U(t, x, y, z)$ is the speed of the walls, depending on the speed of rotation of the cylindrical blades around its own longitudinal axis and the speed of rotation of the wind wheel around the axis of the wind turbine.

\section{The technique of 3-dimensional modeling}

The system of equations (1-9) is solved using Ansys-Fluent package using the finite volume method and the approach of multiple (nested) coordinate systems [12,14]. The entire computational domain is divided into three types of subregions nested into each other, Fig 1 . Subregions of the $1^{\text {st }}$ type (cylinders), built around the working wheels of the wind wheel and rotating at the speed of the working cylinders (1); a subregion of the $2^{\text {nd }}$ type (cylinder) built around the wind wheel minus the cylindrical subregions of the 1 st type (2); type $3^{\text {rd }}$ subdomain (sphere) surrounding the type 2 nd subdomain minus its (3), Fig.1.

The radius of the outer spherical subregion (3) is assumed to be $10 \mathrm{~m}$, the cylindrical subregion (2) has a radius of $2 \mathrm{~m}$ and a height of $0.5 \mathrm{~m}$. The cylindrical subregions (1) have a radius of $0.15 \mathrm{~m}$ and a height of $1.06 \mathrm{~m}$. A finite-volume mesh constructed in subregions 1 -th type, consists of hexagonal cells, and in subdomains of the 2nd and 3rd type - of tetragonal cells.

The grid view in the $\mathrm{z}=0$ plane is shown in Figure 2. The grid is shown in the XY plane, section of the region $\mathrm{Z}=0$. The total number of cells is 742858 .

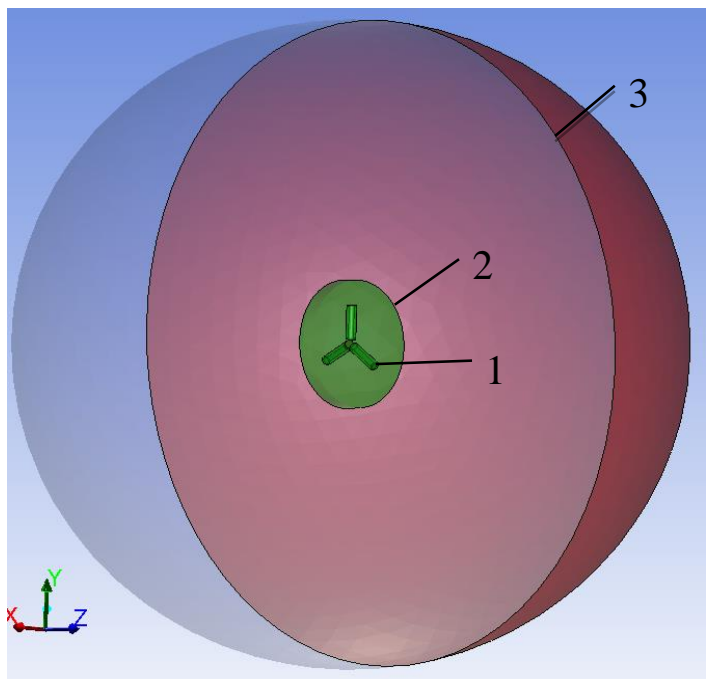

Fig.1. Computing area.

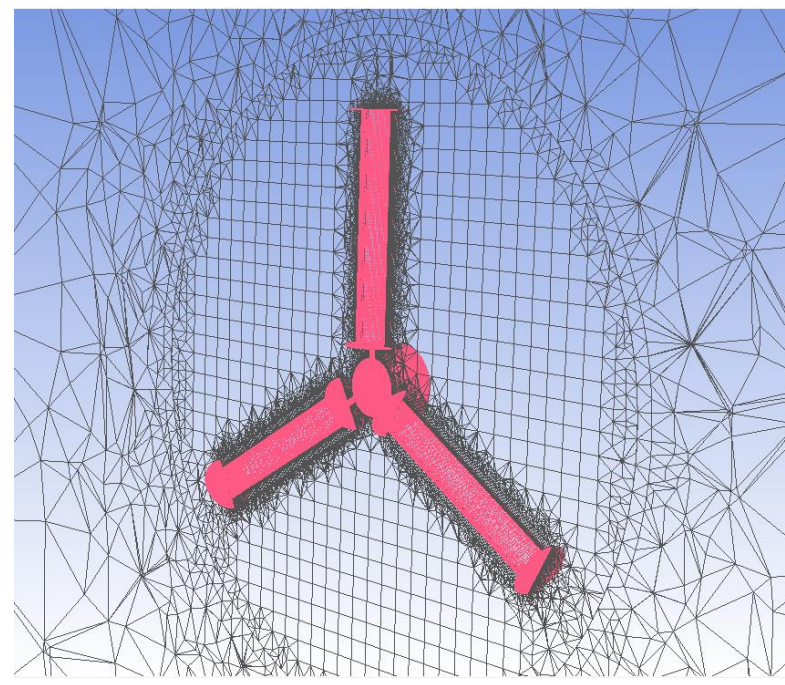

Fig.2. Finite volume mesh

\section{Discussion of results}

\subsection{Modeling the flow field near the wind wheel surface.}

The second-order up flow flow difference scheme in space was used to approximate the convective terms of the system of equations (1-2). The central-difference scheme was used to approximate second-order derivatives. To match the pressure field and the velocity field, the SIMPLE scheme was used $[12,14,15]$. Time derivatives were resolved with a second order of accuracy.

In Fig.3. the longitudinal velocity distributions in the vicinity of a moving wind wheel (plane $\mathrm{z}$ $=0$ ) are shown for various cylinder speeds at a flow rate $\mathrm{V}=10 \mathrm{~m} / \mathrm{s}$. At low speeds of the 
cylinders rotation the flow field is close to symmetrical with respect to three planes of symmetry passing along the axes of the cylinders (Fig.3a), while higher speeds of rotation of the cylinders lead to deformation of the longitudinal velocity field (Fig.3.c). The field is longitudinal speed preserves symmetry when turning $120^{\circ}$ relative to the $\mathrm{z}$ axis.

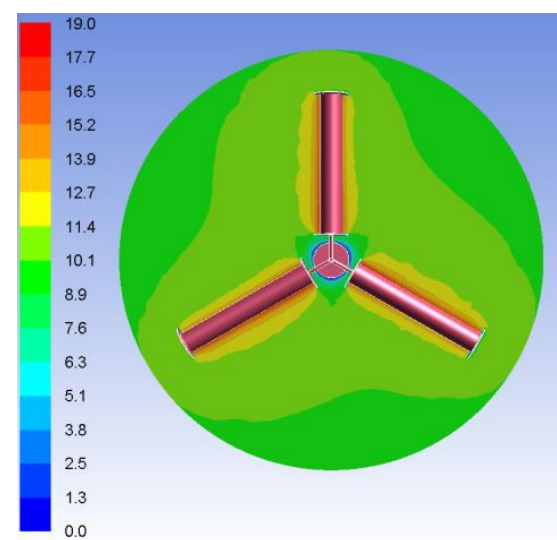

a

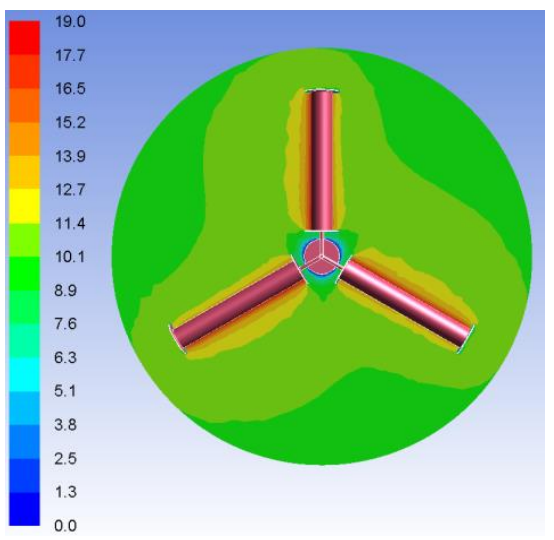

b

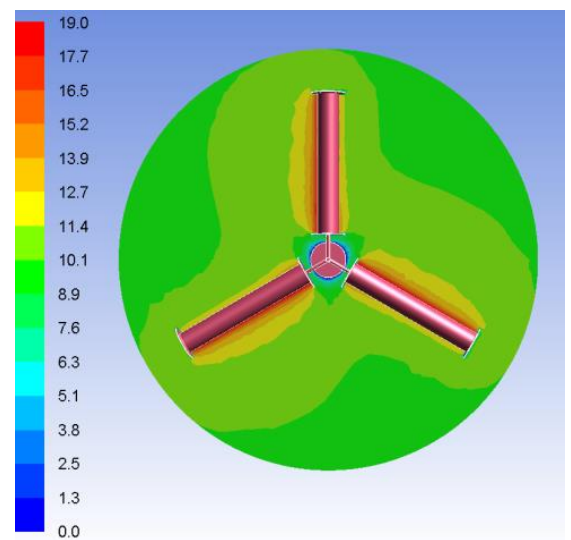

c

Fig. 3. The distribution of the longitudinal velocity $u$ in the vicinity of a movable wind wheel at different speeds of rotation of a cylindrical blade $\mathrm{n}=:$ a) $300 \mathrm{rpm}$; b) $500 \mathrm{rpm}$; ) $700 \mathrm{rpm}$.

Free airflow velocity $\mathrm{V}=10 \mathrm{~m} / \mathrm{s}$.

The deformation of the flow field occurs due to an increase in the velocity of the air flow on one side of the cylinder and a decrease in the flow velocity on the other side, caused by the rotation of the cylinders around their axes in the positive direction (counter clock wise).

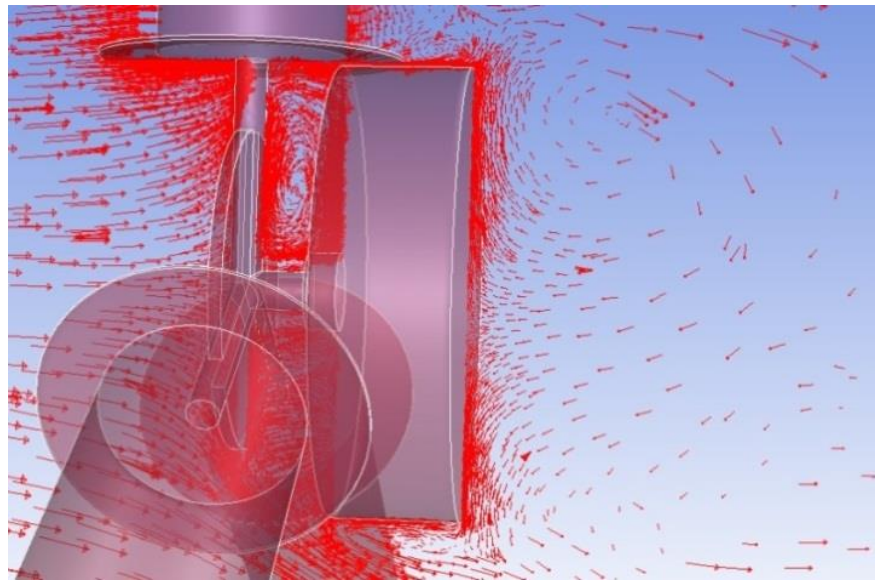

Fig. 4. The velocity field in the plane at the base of the blade, $\mathrm{x}=0$ (plane $\mathrm{YZ}$, cross section $\mathrm{x}=0$ ).

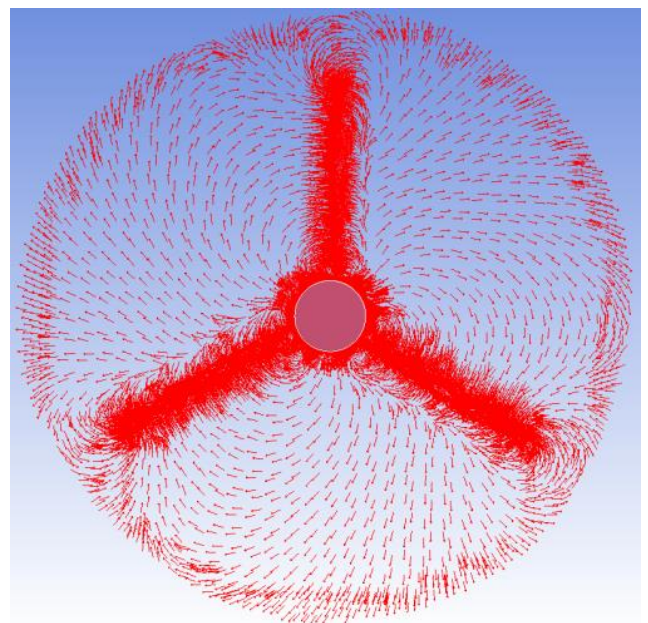

Fig. 5. The flow pattern in the plane $\mathrm{z}=0.15 \mathrm{~m}$.

You can see projections of velocity vectors onto the $\mathrm{x}=0$ plane in the vicinity of the central disk of the wind wheel, Fig.4. It is seen that vortex zones are formed behind the central disk, in which the flow unfolds towards the main flow. The flow pattern in this region is qualitatively the same when the cylinder rotation speed is changing in the range from $300 \mathrm{rpm}$ to $700 \mathrm{rpm}$.

Figure 5 shows the projections of normalized velocity vectors onto the plane $\mathrm{z}=0.15 \mathrm{~m}$ for a movable wind wheel with cylinders rotating around their axes at a speed of $500 \mathrm{rpm}$. It can be seen that the air movement has a complex character: in the vicinity of the center of the wind wheel, the flow is swirling clockwise, and on the periphery, the air moves in a direction close to radial, while in the vicinity of the ends of the cylinders the air flows counter clock wise. 


\subsection{Modeling of aerodynamic characteristics.}

This section presents the results of the calculation of the time-averaged values of the drag force and the lifting force acting on one cylinder, and their coefficients, obtained for a movable wind wheel. When solving the system of equations of conservation of mass and conservation of momenta, both viscous and turbulent components were taken into account.

Averaging over time $t$ was carried out according to the formula:

$$
\langle f\rangle=\frac{1}{T} \int_{0}^{T} f(t) d t
$$

$\mathrm{T}$ is the averaging period, the value of the time interval varies from $3 \mathrm{~s}$ to $5 \mathrm{~s}$.

This duration is much longer than the period of rotation of the cylinders around its own axis of $0.02 \mathrm{~s}$ even at the lowest rotation speed of $300 \mathrm{rpm}$.

In Fig. 6 are shown the results of the aerodynamic characteristics calculating of a wind turbine with rotating cylindrical blades that rotate around axis with speed of $300 \mathrm{pm}, 500 \mathrm{rpm}, 700 \mathrm{rpm}$. An analysis of numerical calculations shows that the drag force coefficient $C_{x}$ value for the same Reynolds number decreases with increasing speed of rotation of the cylinders around its own axis, as well as the magnitude of the lift force coefficient $C_{y}$. To calculate these coefficients, well-known standard formulas were used.

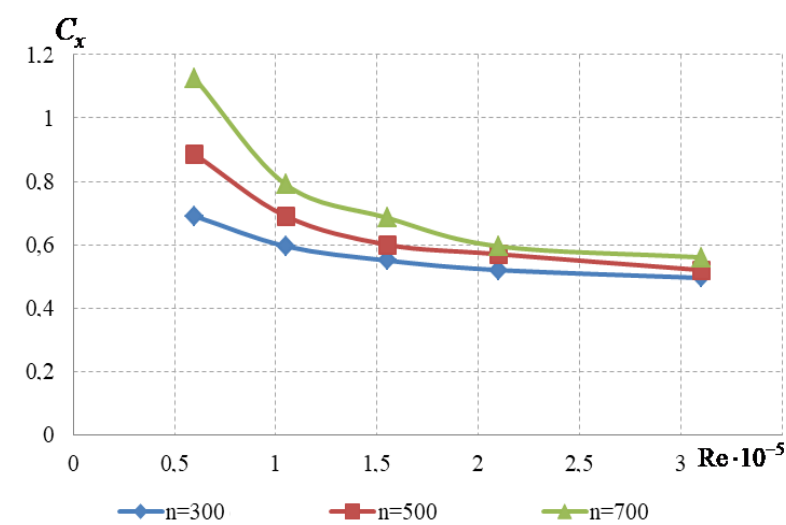

a

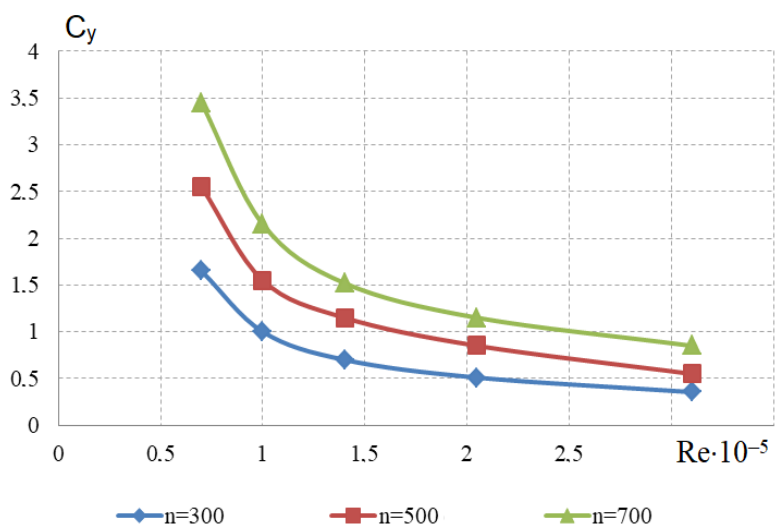

b

Fig.6. Dependence of the aerodynamical coefficients on the air flow speed at different rotation speeds of the cylindrical blade: a) - drag force coefficient; b) - lifting force coefficient.

But the rotation speed of the cylinders leads to an increase in the drag force of the wind wheel to the incoming flow according to a law close to linear, while with increasing flow velocity, the drag force increases according to the quadratic law. The detected changes in the dynamics of the aerodynamic parameters of a model wind turbine as a function of the speed of the incoming air flow depending on the rotational speed of cylindrical blades confirm the influence of the Magnus effect.

It should be noted that the geometric model of the 3-bladed wind turbine was created in the Gambit package, which has powerful capabilities and provides high accuracy of calculations with an error of less than $1 \%$.

\section{Conclusion}

The technique for building a CAD model of a three-bladed wind turbine and simulation of its flow in the ANSYS CFX and ANSYS FLUENT software package had been developed. As a result of three-dimensional modeling, some features have been established in the aerodynamics of the flow around a wind turbine with three rotating cylinders in a turbulent air flow. The results of these studies show that at low flow rates, a rotating cylinder has relatively higher lift ratios, and the effect 
increases with increasing turbulence intensity. The obtained simulation results of aerodynamic parameters are in satisfactory agreement with the experimental results. At this stage of the simulation, the action of the Magnus effect on the flow aerodynamics has been detected. So, rotating cylinders can be effectively used as a power element of a wind turbine under choosing the optimal conditions for the flow around.

\section{REFERENCES}

1 World Renewables 2019. Global Status Report. https://www.ren21.net/reports/global-statusreport/

2 Karatayev M., Hall S., Kalyuzhnova Y., Clarke M. Renewable Energy Technology Uptake in Kazakhstan: Policy Drivers and Barriers in a Transitional Economy. Renewable and Sustainable Energy Reviews. 2016, Vol. 66, pp. 120-136.

3 Shadrina, E. Renewable Energy in Central Asian Economies: Role in Reducing Regional Energy Insecurity. Tokyo. 2019. Available at: www.adb.org/publications/renewable-energy-central-asianeconomies

4 Mukhamediyev I.R., Mustakayev R., et al. Multi-criteria spatial decision making system for renewable energy development support in Kazak-hstan. IEEE Access. 2019, Issue 7, pp.122275-122288.

5 Martin O. L. Hansen L. Aerodynamics of Wind Turbines. London, Sterling, VA, 2008, 208 p.

6 Sakipova S.E., Jakovics A., Gendelis S. The Potential of Renewable Energy Sources in Latvia. Latvian Journal of Physics and Technical Sciences. Riga, 2016, Vol.53, Issue 1, pp. 3 - 12.

7 Kussaiynov K., Sakipova S.E., Tanasheva N.K., Kambarova Zh.T., et al. Wind turbine based on the Magnus effect. Innovative patent №30462 of 23.09.2015.

8 Yershina A.K., Yershin Sh.A., Yershin Ch., Manatbayev R.K. Wind motor. Kazakhstan Republic Patent, No.31662. 2016, Bulletin 15, 5 p.

9 Yershina A.K., Manatbayev R.K., Sakipova S.E. Some design features of the carousel type wind turbine Bidarrieus. Eurasian phys. tech. j. 2019, Vol.16, No. 2(32). - P.63- 67.

10 Kayan V. P., Kochin V. A., Lebid O. G. Studying the Performance of Vertical Axis Wind Turbine Models with Blade Control Mechanism. Intern. Journal of Fluid Mechanics Research. 2009, Vol.36, Issue 2, pp. $154-165$.

11 Sakipova S.E., Tanasheva N.K., Kussaiynova A.K. Study of aerodynamics of a two-bladed wind turbine with porous-surfaced cylindrical blades. Eurasian phys. tech. j. 2017, Vol.14, No.28, pp.120 - 124.

12 Sakipova S.E., Tanasheva N.K. Modeling aerodynamics of the wind turbine with rotating cylinders. Eurasian phys. tech. j., 2019, Vol.16, No. 1(31), pp. 88 - 93.

13 Miau J.J., Tsai J.H., Hsu X.Y., et al. On critical transition of flow over a circular cylinder roughened by textile materials. AIP Conference Proceedings 2027. 2018, pp. 020004-1 - 020004-10. doi: 10.1063/1.5065082

14 ANSYS Fluent software. Available at: https://www.ansys.com/products/fluids/ansys-fluent

15 Computer modeling of 3D-models of aviation equipment and engineering calculations. Available at: http://www.ipmce.ru/custom/vsop/themes/3dmodel/ 